$\operatorname{Sir}$

\section{Ischaemic maculopathy in japanese encephalitis}

Japanese encephalitis is caused by Japanese encephalitis virus (JEV), which predominantly affects the thalamus, basal ganglia, substantia nigra, pons, cerebellum, and cerebral cortex. ${ }^{1}$ Now we report a patient with JEV-related ischaemic maculopathy.

\section{Case report}

A 53-year-old woman had fever and headache for 4 days. Elevated blood pressure, limbs weakness, neck stiffness, and progressive disturbance of consciousness were also noted. On the fifth day, a tonic-clonic seizure episode occurred, and Glasgow Coma Scale score dropped to 3 soon afterwards. Magnetic resonance imaging showed bilateral thalamic and midbrain lesions. Serum and cerebrospinal fluid examination revealed rising titre of JEV-specific immunoglobulin $\mathrm{M}$ antibodies. Polymerase chain reaction analysis of cerebrospinal fluid also confirmed JEV infection. Tests for other viral infections, such as herpes simplex virus, varicella-zoster virus, cytomegalovirus, and human immunodeficiency virus, were all negative.

With supportive care in the intensive care unit, she gradually recovered from the comatose status 6 days later, but profound comprehensive and expressive difficulties persisted for 1 month. When she could express herself more clearly, blurred vision in the right eye was noted. Ophthalmic examination revealed a visual acuity of counting fingers in the right eye and $20 / 25$ in the left eye. Slit-lamp examination of anterior segments was unremarkable. There was no vitreal cell. Funduscopy examination showed a highly elevated, yellowish white macula with surrounding retinal haemorrhages in the right eye and retinal haemorrhage in the temporal macula of the left eye. Fluorescein angiography of the right eye revealed a large nonperfusion area of the macula and leakages around the non-perfusion retina. Prominent leakages on temporal macula were also demonstrated in the left eye (Figure 1). Reviewing the T2-FLAIR magnetic resonance imaging

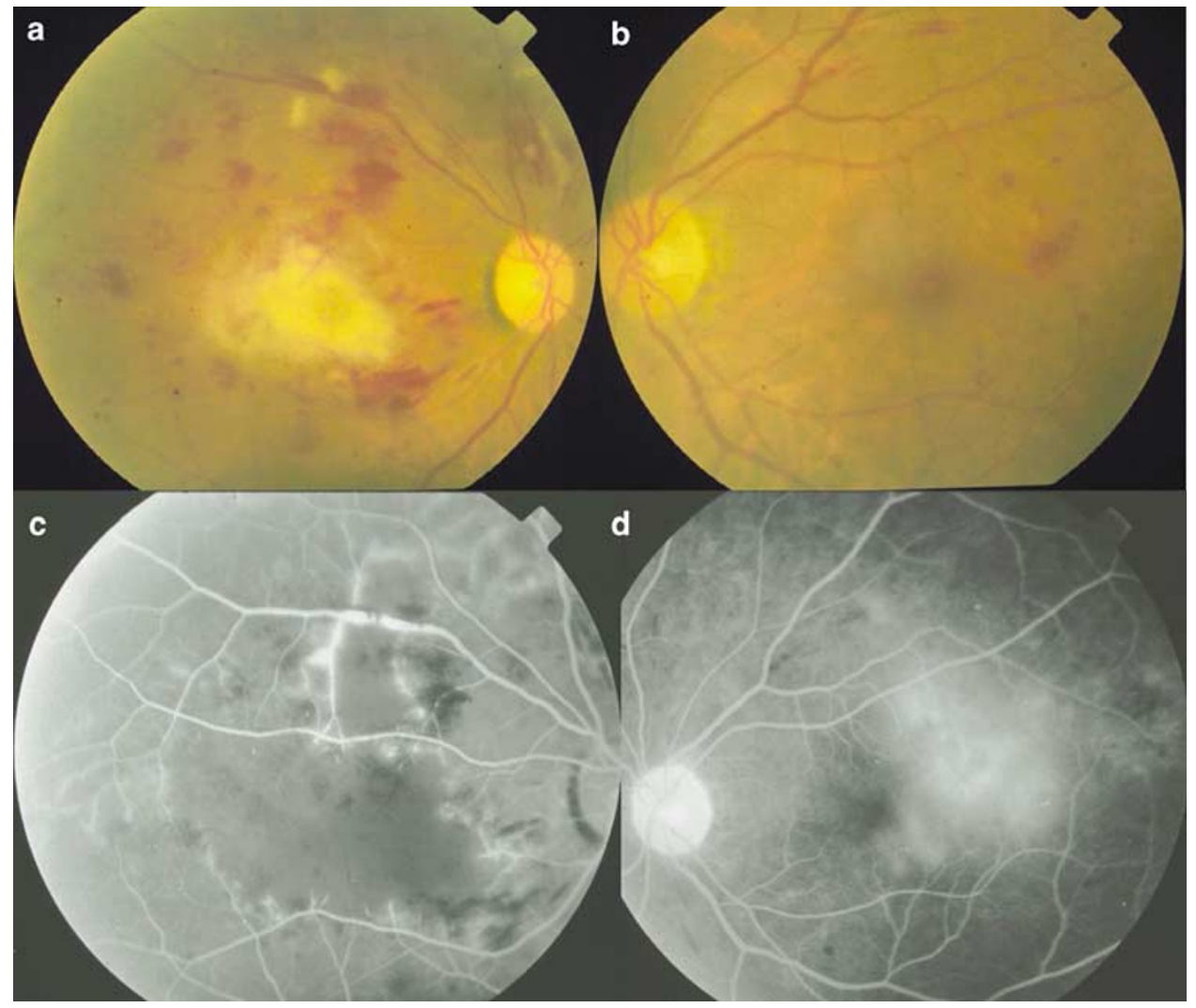

Figure 1 Ischaemic maculopathy in this JEV-infected patient noted upon recovery from consciousness disturbance. (a) Colour photograph of the right eye showed highly elevated, yellowish white macula with surrounding retinal haemorrhages. (b) Photograph of the left eye showed haemorrhages in the temporal macula. (c) Fluorescein angiography of the right eye revealed a large non-perfusion area of the macula, and fluorescein dye leakages around the nonperfusion area. (d) Fluorescein angiography of the left eye showed prominent leakages on temporal macula. 

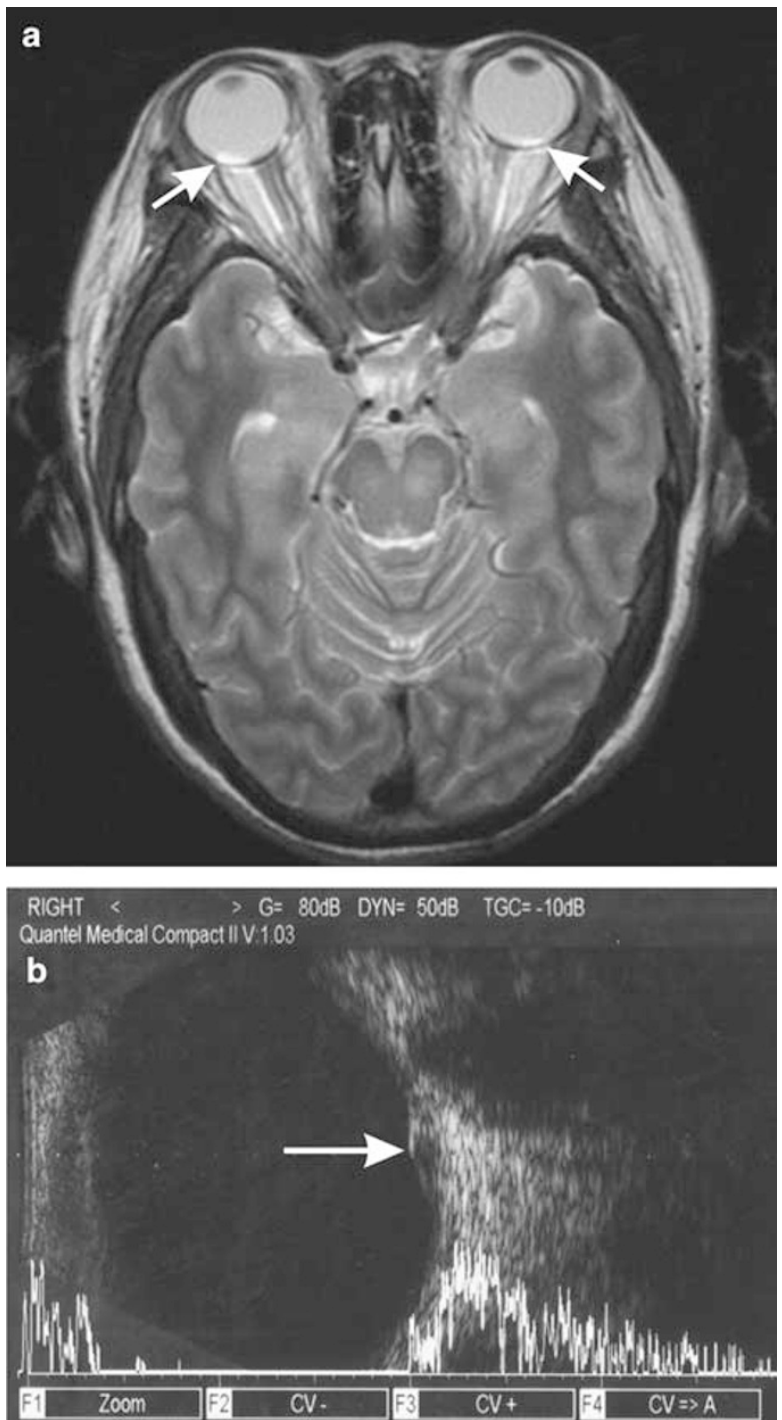

Figure 2 (a) T2-FLAIR magnetic resonance imaging on loss of consciousness showed hyperintensity of bilateral macular areas (arrows). (b) B-scan ultrasonography on recovery of consciousness demonstrated the elevation of right eye macula (arrow).

found hyperintensity of the corresponding areas. B-scan ultrasonography also demonstrated elevation of macula in the right eye (Figure 2).

Argon laser photocoagulation was applied to the ischaemic retina outside the arcade. During the 1-year follow-up, the whitening and elevation of the macula subsided, the area of nonperfusion decreased, and the leakages resolved. However, the visual acuity remained counting fingers in the right eye.

\section{Comment}

Previous pathological studies in JEV-infected brains revealed extensive areas of congestion, perivascular cuffing, small haemorrhages, and thrombus

formation in the acute stage (within 1 week), and fibrous thickening of vascular walls and post-inflammatory organization in the chronic stage (after several weeks to months). ${ }^{2}$ SPECT studies using ${ }^{99 \mathrm{~m}} \mathrm{Tc}$ revealed hypoperfusion of the involved areas, ${ }^{3}$ which further indicates the ischaemic nature of JEV infection. Besides, it has been shown that a JEV-induced cytokine, macrophage-derived neutrophil chemotactic factor, alters the integrity of the blood-brain barrier and results in leakage. ${ }^{4}$ These findings might explain the severe macula ischaemia in the right eye and leakages in both eyes in our patient.

Similar ischaemic maculopathy has been reported in HIV-infected individuals. ${ }^{5}$ Widespread retinal capillary closure followed by progressive closure of the large retinal vessels was proposed. ${ }^{6}$ Although reduced macular leucocyte density or velocity in HIV-infected individuals has been shown, ${ }^{7,8}$ the pathogenesis remains unknown. In our patient, there was no vitreal cell, perivascular cuffing, or sheathing. Fluorescein angiography showed extensive capillary closure of the macula, with mild leakage around the nonperfusion area. There was no evident delay in arterial or venous filling. We therefore favoured the diagnosis of ischaemic maculopathy. However, the mechanism underlying ischaemic maculopathy in JEV-infected patients needs further investigation.

This appears to be the first case report of ocular fundus presentation in Japanese encephalitis. In our patient, the MRI performed on disturbance of consciousness already showed hyperintensity on bilateral macula areas, but was not noted until ophthalmic consultation. It was neglected at first because of disturbance of consciousness of the patient and lack of report on ocular manifestation in Japanese encephalitis. We therefore recommend a notice at ocular areas on MRI and ocular fundus examination in Japanese encephalitis. Besides, the visual recovery was not good with conservative follow-up in this patient. Other modalities or more aggressive intervention, such as anticoagulants, antiplatelets, or fibrinolytic agents, may be considered while facing similar situation.

\section{References}

1 Kalita J, Misra UK. Neurophysiological changes in Japanese encephalitis. Neurol India 2002; 50: 262-266.

2 Miyake M. The pathology of Japanese encephalitis. A review. Bull World Health Organ 1964; 30: 153-160.

3 Kalita J, Das BK, Misra UK. SPECT studies of regional cerebral blood flow in 8 patients with Japanese encephalitis in subacute and chronic stage. Acta Neurol Scand 1999; 99: 213-218. 
4 Mathur A, Khanna N, Chaturvedi UC. Breakdown of blood-brain barrier by virus-induced cytokine during Japanese encephalitis virus infection. Int J Exp Pathol 1992; 73: 603-611.

5 Cunningham Jr ET, Levinson RD, Jampol LM, Engstrom Jr RE, Lewis H, Holland GN. Ischemic maculopathy in patients with acquired immunodeficiency syndrome. Am J Ophthalmol 2001; 132: 727-733.

6 Roth DB, McCabe CM, Davis JL. HIV-related occlusive vasculitis. Arch Ophthalmol 1999; 117: 696-698.

7 Lim MC, Cumberland WG, Minassian SL, Ransome SS, Cornish MJ, Terry BG et al. Decreased macular leukocyte velocity in human immunodeficiency virus-infected individuals. Am J Ophthalmol 2001; 132: 711-719.

8 Dejaco-Ruhswurm I, Kiss B, Rainer G, Krepler K, Wedrich A, Dallinger $\mathrm{S}$ et al. Ocular blood flow in patients infected with human immunodeficiency virus. Am J Ophthalmol 2001; 132: 720-726.

\section{S-T Fang ${ }^{1}$, S-Y Chu ${ }^{2}$ and Y-C Lee Le, $^{1,4,5}$}

${ }^{1}$ Department of Ophthalmology, Buddhist Tzu Chi

General Hospital, Taiwan, Taiwan

${ }^{2}$ Department of Pediatrics, Buddhist Tzu Chi

General Hospital, Taiwan, Taiwan

${ }^{3}$ Department of Ophthalmology, National Taiwan

University Hospital, Taiwan, Taiwan

${ }^{4}$ The Department of Medicine, Tzu Chi University, Taiwan, Taiwan

${ }^{5}$ Graduate Institute of Medical Sciences,

Tzu Chi University, Taiwan, Taiwan

Correspondence: Y-C Lee,

Department of Ophthalmology,

Buddhist Tzu Chi General Hospital, 707,

Section 3, Chung Yang Road, Hualien,

Taiwan 970, Taiwan

Tel: + 88638561825 ext 3253;

Fax: +88638578179

E-mail: derrick@url.com.tw

Financial support: none

Proprietary interest statement: none

Eye (2006) 20, 1439-1441. doi:10.1038/sj.eye.6702301; published online 24 February 2006
Sir,

Ultrasound biomicroscopy demonstrating etiology of a spontaneous filtering bleb

We report a case of delayed onset spontaneous conjunctival filtering bleb appearing many years after penetrating trauma. The bleb fistula site was identified on ultrasound biomicroscopy (UBM), but was not apparent on clinical examination.

\section{Case report}

A 68-year-old man presented after noting a growth on the nasal aspect of his right eye conjunctiva 1 month ago. The growth did not increase in size, and was not associated with ocular or visual symptoms. He reported history of penetrating injury to that eye with glass during adolescence that required surgical repair at the time.

In the right eye, best-corrected visual acuity was 20/25 applanation tonometry was $19 \mathrm{~mm} \mathrm{Hg}$. Biomicroscopy revealed a conjunctival cystic bleb nasally (3-4:30 $\mathrm{O}^{\prime}$ clock) in proximity to a corneal-limbal scar (Figure 1). The anterior chamber was shallow nasally with iridocorneal adhesions and a distorted pupil. The lens showed mild nuclear sclerosis. Gonioscopy of the nasal angle revealed a localized 'dimpled' scarring and no cleft (Figure 2). Ultrasound biomicroscopy of the bleb site showed scleral fistulization from the posterior aspect of the bleb to the ciliary body (Figure 3). The rest of his ophthalmic evaluation, including the left eye examination was within normal limits.

\section{Comment}

Spontaneous filtering conjunctival blebs are rare and can be observed in patients with systemic or ocular abnormalities like Terrien's marginal degeneration, Axenfeld syndrome, or familial craniofacial

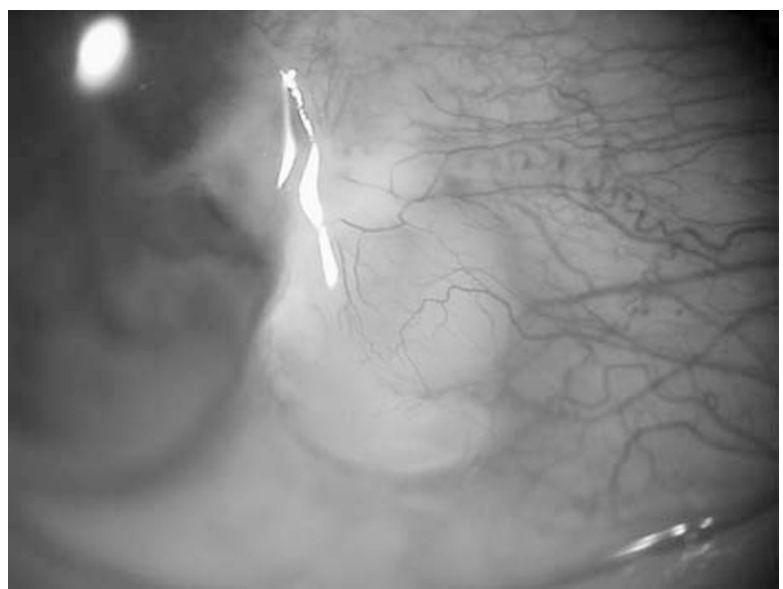

Figure 1 A localized conjunctival filtering bleb nasally in the right eye. 